\section{IDDF2019-ABS-0215 ANTIMICROBIAL PROPHYLAXIS AFTER HEPATIC RESECTION FOR HEPATOCELLULAR CARCINOMA: A PROPENSITY SCORE MATCHING STUDY}

${ }^{1}$ Zebin Chen*, ${ }^{2}$ Shuling Chen, ${ }^{3}$ Ruiming Liang, ${ }^{3}$ Zhenwei Peng, ${ }^{3}$ Sui Peng. 'Department of Liver Surgery, The First Affiliated Hospital of Sun Yat-sen University, China; ${ }^{2}$ Division of Interventional Ultrasound, The First Affiliated Hospital of Sun Yat-sen, China; ${ }^{3}$ Clinical Trials Unit, The First Affiliated Hospital of Sun Yat-sen University, China

\subsection{6/gutjnl-2019-IDDFabstracts.287}

Background Hepatic resection (HR) is one of the curative treatments for hepatocellular carcinoma (HCC) and infection is a common complication after HR. For clean-contaminated surgery, the guideline recommends only preoperative prophylactic antibiotics; however, antibiotics are frequently used postoperatively in reality. We investigate whether postoperative prophylactic antibiotics can reduce infection for HR.

Methods From January 2009 to December 2017, patients whom underwent $\mathrm{HR}$ for Barcelona Clinic Liver Cancer (BCLC) stage 0/A/B HCC in three tertiary referral centers were included in the study. Patients were divided into two groups according to whether they used prophylactic antibiotics postoperatively or not. One group did not use antibiotics after HR (group A), and the other group did (group B). The infection rates were estimated and compared between the two groups before and after matching the propensity scores.

Results In total, 1049 patients were included, with 273 patients in group A and 776 patients in group B. The median days of postoperative antibiotic use in group B is 5.0 days (interquartile range [IQR], 3.0-7.0 days). The infection rate was $27.5 \%$ and $6.8 \%$ in group $\mathrm{A}$ and $\mathrm{B}$, respectively $(P<0.001)$. Prothrombin time $>14$ seconds (OR 2.05, 95\% CI 1.10-3.83), albumin < 35 g/L (OR 1.89, 95\% CI 1.013.54 ) and postoperative prophylactic antibiotics (OR 0.20, 95\% CI 0.14-0.30) were independently associated with infection. After matching the propensity scores, the infection rates in the group $\mathrm{A}(\mathrm{n}=260)$ and $\mathrm{B}$ were $28.1 \%$ and $8.5 \%$, respectively $(P<0.001)$.

Conclusions Postoperative prophylactic antibiotics can reduce the incidence of infection after HR for HCC.

\section{IDDF2019-ABS-0217 ASSESSMENT OF HEPATIC STEATOSIS IN PATIENTS WITH ANOREXIA NERVOSA USING QUANTITATIVE ELASTOGRAPHY}

Taketo Nishina*, Hiroaki Haga, Kei Mizuno, Kyoko Hoshikawa, Kazuo Okumoto, Takafumi Saito, Yoshiyuki Ueno. Division of Gastroenterology, Yamagata University Faculty of Medicine, Yamagata, Japan

10.1136/gutjnl-2019-IDDFabstracts.288
Background The number of patients with anorexia nervosa (AN) is increasing as society changes. Approximately 30\% of patients with AN have mild liver injury. A part of patients with AN has been reported to present fatty liver change despite of their extremely low body mass index (BMI). Recently, quantification of hepatic fat content is available by FibroScan using a controlled attenuation parameter (CAP) software. In this study, we conducted the FibroScan measurement in patients with AN to assess their steatosis.

Methods Eighteen patients hospitalized with a diagnosis of AN were enrolled. Clinical parameters such as age, gender, BMI, as well as routine laboratory data were evaluated. We also assessed their hepatic steatosis by using a CAP software. We defined the level of CAP over $200(\mathrm{~dB} / \mathrm{m})$ as cut off value for hepatic steatosis, and compared these clinical parameters among non-fatty (non-FL) group (9 patients, levels of $\mathrm{CAP}<$ 200) and fatty liver (FL) group (9 patients, levels of CAP>200).

Results All the enrolled subjects were female with a median age of $33.5(13-62)$ years and BMI $13.4 \mathrm{~kg} / \mathrm{m}^{2}$. Their mean

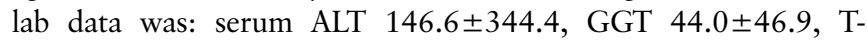
chol 194.2 \pm 44.3 , TG $72.4 \pm 33.5$, the mean levels of CAP $214.6 \pm 54.4$, hepatic stiffness $5.7 \pm 1.6 \mathrm{kPa}$. Ten patients $(55 \%)$ was positive for LK contrast. After medical treatment for 4 weeks, the levels of CAP in FL group decreased $248 \pm 55.6$ to 207.5 $\pm .29 .1 \quad(p=0.042)$, and of those in non-FL group increased $176.7 \pm 9.9$ to $190.1 \pm 31.5$. The changes of serum Tchol and TG in FL group were: $172.8 \pm 35.8$ to $210.6 \pm 38.2$ $(p=0.033)$, and $63.1 \pm 15.9$ to $84.5 \pm 33.8$, whereas those in non-FL group were: $215.5 \pm 41.7$ to $185.2 \pm 41.7$, and 81.7 \pm 42.6 to $59.5 \pm 28.9$, respectively.

Conclusions After 4 intervention, the levels of CAP in FL group decreased, and sonographic hepatic steatosis was improved. Further studies are feasible to clarify the mechanism of steatosis in patients with AN.

\section{IDDF2019-ABS-0220 LONG-TERM OUTCOMES OF UTILIZING EXTENDED CRITERIA DECEASED DONORS IN LIVER TRANSPLANTATION - AN AUSTRALIAN 12-YEAR COHORT STUDY}

Keval Pandya*, Vinay Sastry, Mara Panlilio, Claire West, Susan Virtue, Mark Wells, Michael Crawford, Carlo Pulitano, Simone Strasser, Geoff McCaughan, Avik Majumdar, Ken Liu. Australian National Liver Transplant Unit, Royal Prince Alfred Hospital, Sydney, Australia

\subsection{6/gutjnl-2019-IDDFabstracts.289}

Background The need for liver transplantation (LT) is increasing worldwide; however, organ shortage presents a major limitation. Use of extended criteria donors (ECDs) has been one
A

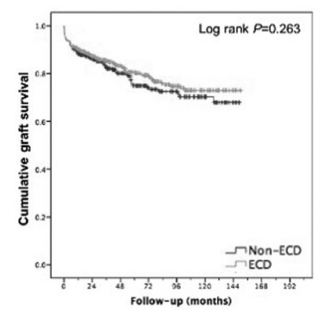

B

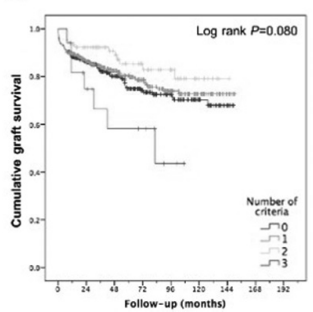

C

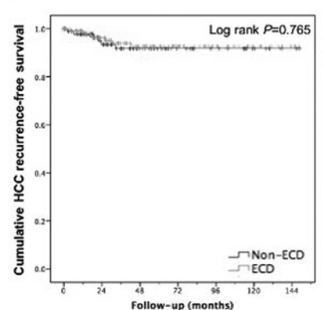

